\title{
Saúde da família: desafios no processo de reorientação do modelo assistencial
}

\author{
Family health: challenges in the reorientation process of the assistance model
}

Salud de la familia: desafíos en el proceso de reorientación del modelo de atención

\section{Glauce Dias da Costa', Rosângela Minardi Mitre Cotta', Maria de Lurdes da Silva Marques Ferreiral", José Roberto Reis', Sylvia do Carmo Castro Franceschinil \\ 'Universidade Federal de Viçosa. Departamento de Enfermagem. Viçosa, MG \\ "Universidade Estadual Paulista. Botucatu, SP}

Submissão: 12/02/2008

Aprovação: 24/1 1/2008

\section{RESUMO}

O Programa Saúde da Família (PSF) constitui-se em uma estratégia de reformulação do modelo assistencial de saúde no Brasil. Este estudo tem como objetivo refletir sobre os desafios desta estratégia Quanto às práticas de saúde no contexto da atenção primária. Foi realizado um estudo bibliográfico, 90 artigos foram selecionados, sendo 50 considerados de maior interesse. Após Quatorze anos de existência, surgem Questionamentos Quanto ao papel do PSF, suas estruturas continuam permeáveis ao modelo hegemônico Que corrompe o processo de trabalho cotidiano. Mudanças nas práticas de saúde, especialmente, no Que tange a produção de cuidados, são necessárias e este processo de reconstrução implica muito labor. No entanto, possibilita a construção de práticas de saúde mais solidárias, acolhedoras e conseqüentemente mais resolutivas.

Descritores: Saúde da família; Atenção primária; Programa Saúde da Família.

\begin{abstract}
The Family Health Program (PSF) constitutes a strategy for reformulating the health assistance model in Brazil. This study was carried out to ponder over the challenges of this strategy concerning to health practices in the primary care context. After existing for more than twelve years, there appear Questionings related to the paper of PSF in the reformulation of the way to think and promote health, and their structures remain permeable to the traditional hegemonic model that corrupts the daily work process. Some changes and reconstruction are necessary in the health practices concerning to care production based on light, relational and integrate technologies that are centered on users' needs. Although the deconstruction/reconstruction process implicates much labor, the advantage in overcoming the challenges faced by PSF rather leads to the transformation of reality and to construction of health practices that are solidary, welcoming, and resolvent.
\end{abstract}

Descriptors: Family health; Primary health care; Family Health Program.

\section{RESUMEN}

El Programa de la Salud Familia (PSF) se constituye en una estrategia de reformulación del modelo de salud en Brasil. Este estudio tiene como objetivo reflejar sobre los retos del PSF en lo Que se refiere a las prácticas de salud en el contexto de la atención primaria. Después de más de doce años de existencia del PSF surgen cuestionamientos en cuanto a la importancia del PSF en la reformulación en la forma de pensar y hacer salud, siendo Que sus estructuras persisten permeables al modelo tradicional hegemónico Que pervierte el proceso de trabajo cotidiano. Cambios en las prácticas de salud son necesarios en relación a la producción de la atención, basadas en tecnologías leves, relacionales, integradas, y centradas en las necesidades de los usuarios. El proceso de reconstrucción implica en arduo trabajo, mas la ventaja de la superación de los retos enfrentados por el PSF lleva a la transformación de la realidad y la construcción de prácticas de salud solidarias, acogedoras y consecuentemente más efectivas y de resolución.

Descriptores: Salud de la familia; Atención primaria de salud; Progrma de Salud Familiar. 


\section{INTRODUÇÃO}

Após conquistas inegáveis do ponto de vista jurídico legal do sistema de saúde brasileiro, tem-se, atualmente, como Questão central à reformulação das organizações e estabelecimentos sanitários. Assim, busca-se criar condições para Que, de forma permanente, o sistema de saúde, aproxime-se mais dos indivíduos, das famílias e das comunidades, torne-se mais humanizado, solidário e, sobretudo, mais resolutivo ${ }^{(1)}$.

O Programa de Saúde da Família (PSF), criado em 1994 pelo Ministério da Saúde (MS), foi formulado como uma estratégia para transformação do modelo de atenção à saúde no Brasil na busca de provocar reflexões e mudanças nas instituições, nos padrões de pensamento e comportamento dos profissionais e cidadãos brasileiros ${ }^{(2,3)}$. Trata-se, portanto, de uma estratégia que envolve a comunidade, por meio dos Agentes Comunitários de Saúde (ACS) e coloca as equipes multiprofissionais mais perto dos domicílios, das famílias e das comunidades.

Esta proposta luta para substituir a forma de pensar e praticar saúde, transformando o tradicional modelo sanitário brasileiro, médico, medicamentoso, curativo e individual Que tem no hospital o lócus de solução de todo e Qualquer problema de saúde, em um modelo de saúde coletivo, multi e interprofissional, centrado na família e na comunidade ${ }^{(4,5)}$. O desafio Que se coloca é a transformação da atenção sanitária centrada no procedimento em uma atenção centrada no usuário ${ }^{(6)}$.

No entanto, depois de mais de doze anos de sua existência, surgem Questionamentos Quanto a prática cotidiana dos PSF's na reformulação da forma de pensar e fazer saúde, sendo Que, não raras vezes, suas estruturas continuam permeáveis ao modelo tradicional biomédico Que corrompe o processo de trabalho cotidiano $^{(7)}$. Há portanto, uma ação "sanguessuga", pois o modelo Que necessita ser alterado suga as forças desta estratégia de reformulação em sua arma mais poderosa Que são as práticas de saúde. Práticas de saúde ainda influenciadas pelo tradicional paradigma flexneriano cujo conceito de saúde é tomado em sua negatividade, ao pensar a saúde enQuanto a ausência de doença, dor e morte, tendo a organização dos serviços medicamente definida.

Descontruir o modelo de saúde vigente é enfrentar o desafio de construir novas bases para o desenvolvimento de novas práticas sanitárias. Neste sentido, é sob esta perspectiva, Que este estudo se propõe a uma análise e compreensão das possibilidades e limites do PSF como estratégia de reorientação do modelo assistencial e como produtor de cuidados.

\section{MÉTODO}

Realizou-se uma revisão minuciosa da literatura, a partir das bases de dados LILACS e MEDLINE, consultou-se também as bases de dados de saúde pública de difusão nacional e internacional, entre elas Scientific Electronic Library (SCIELO), periódicos da CAPES, documentos e publicações do Ministério da Saúde e da Organização Panamericana de Saúde (OPAS). Para as consultas nestas bases utilizaram-se os seguintes indexadores: "Atenção Primária", "Atenção Básica", "Programa de Saúde da Família", "Modelo assistencial". Entre os 90 artigos selecionados, 50 foram considerados de maior interesse para o estudo proposto; destes, foram analisados detalhadamente 30 artigos conforme os objetivos delineados neste estudo.

\section{RESULTADOS E DISCUSSÃO}

\section{Necessidade de Reorientação do Modelo Assistencial}

No Brasil, a partir da segunda metade da década de 70, em decorrência da crise do setor saúde (financeira, burocráticoadministrativa e de cobertura) o modelo assistencial Que historicamente predominou em todo país vivencia uma profunda crise, emergindo no âmbito da saúde pública algumas propostas Que visam à transformação das práticas das ações de saúde, objetivando a ampliação da cobertura para a população excluída pela assistência previdenciária ${ }^{(4,5,7)}$.

O processo de redemocratização, na década de 80 , com a ampliação da organização popular e a emergência de novos atores sociais, produziu a ampliação das demandas sobre o Estado e desencadeou um forte movimento social pela universalização do acesso e pelo reconhecimento da saúde como direito universal e dever do Estado. O Movimento da Reforma Sanitária (MRS) reuniu atores diversificados em uma batalha política pelo reconhecimento da saúde como direito social, pela universalização do acesso aos serviços de saúde e pela integralidade da atenção à saúde, na luta mais ampla pela construção da cidadania Que marcou o final do regime militar ${ }^{(8)}$.

A realização da VIII Conferência Nacional de Saúde, em I 986, constitui um marco na história da saúde do Brasil, Que resulta na ampla legitimação dos princípios e da doutrina do MRS. Como conseeüência desse fortalecimento político, o MRS conQuistou a incorporação desses princípios ao texto constitucional de 1988 , bem como a determinação da responsabilidade do Estado na provisão das ações e serviços necessários à garantia desse direito, mediante políticas sociais e econômicas Que visem a redução do risco de doença e de outros agravos e assegurem o acesso universal e igualitário às ações e serviços para a promoção, proteção e recuperação de saúde, garantindo um processo de produção social da saúde ${ }^{(3,4,8)}$.

Conquistadas as bases constitucionais e legais, observa-se, no entanto, Que a operacionalização da reforma no setor saúde, visando a construção de um sistema de serviços de saúde democrático, universal, igualitário e integral, encontrou alguns obstáculos $^{(4)}$. A brutal e rápida diminuição dos investimentos Que permitissem ampliar as condições da oferta e a compressão dos valores de remuneração aos prestadores públicos e privados, teve como resultado a progressiva degradação da Qualidade dos serviços oferecidos $^{(9)}$ somados a uma crise da atenção médica manifestada na desorganização dos hospitais e ambulatórios, em Que se misturam ingredientes perversos: filas, atendimento desumanizado, pacientes nos corredores, mortes desnecessárias, etc $^{(10,11)}$.

Desta forma, seria necessário um processo de construção social de um novo sistema de saúde com mudanças estruturais, tendo como propósito a realização de transformação no modelo assistencial ${ }^{(10)}$. Para a construção do sistema de saúde brasileiro (SUS), é fundamental a reorientação do modelo assistencial vigente e hegemônico no país, pautado em uma atenção médicoassistencial privatista ${ }^{(12)}$. Esse modelo legalmente instituído e 
praticado até 1988 estava estabelecido na lei 6.229/75, criando dicotomias entre curativo e preventivo, individual e coletivo, por meio de práticas assistenciais fortemente centradas em hospitais, restritas aos contribuintes previdenciários ${ }^{(1)}$.

O conceito de saúde, para este modelo de atenção, é tomado em sua negatividade, como ausência de doença, amparado no paradigma flexneriano sob influências da Escola Norte - Americana, estrutura como resposta à prática da atenção médica, técnicoassistencial nos marcos da medicina científica ${ }^{(I)}$. Tendo como referência este olhar sob o processo saúde-doença, tem-se o conceito de doença, a partir da "redução do corpo humano, pensado através da forma e função as Quais se definem por intermédio de ciências como a anatomia e a fisiologia"(13).

De acordo com Canguilhem ${ }^{(14)}$ doença é concebida como alterações concretas do corpo do doente. O corpo é assim, desconectado de todo o conjunto de relações Que constituem os significados da vida, desconsiderando Que a prática médica entra em contato com homens e não apenas seus órgãos e funções.

A lógica desta prática de atenção médica é de Que as doenças devem ser enfrentadas com mais serviços, ou seja, se há mais serviços de atenção disponíveis, a saúde deve melhorar e se esses serviços são universais, as disparidades de saúde devem atenuar$\mathrm{se}^{(10)}$.

Contrastando com esta idéia, de acordo com Paim ${ }^{(15)}$ modelo de atenção ou modelo assistencial "é uma dada forma de combinar técnicas e tecnologias para resolver problemas e atender necessidades de saúde individuais e coletivas. É uma razão de ser, uma racionalidade, uma espécie de lógica Que orienta a ação". Esta concepção do modelo assistencial apresentada por Paim ${ }^{(15)}$ fundamenta a consideração de Que o fenômeno isolado da expansão do número de serviços de saúde não garante a construção de um novo modelo assistencial ${ }^{(12)}$.

Desta forma, impõe-se a construção social de uma nova prática Que visa sistematizar de modo detalhado, a proposta de reorganização do SUS, tendo por base a proposta de vigilância a saúde ou da Produção Social da Saúde ${ }^{(10)}$. Para Teixeira et a ${ }^{(16)}$ esta é concebida ainda em uma perspectiva do movimento sanitário, ou seja, não reduzindo suas ações a uma proposta simplesmente de maior racionalização das ações de saúde pública. A proposta de Vigilância à Saúde supera os modelos assistenciais vigentes implicando uma redefinição do sujeito, do objeto e das formas de organização dos processos de trabalho.

Desde esta perspectiva, na década de 1990, tem inicio a implementação da estratégia do PSF Que, no contexto da política de saúde brasileira deveria contribuir para a construção e consolidação do SUS. A estratégia do PSF traz no centro de sua proposta a expectativa relativa à reorientação do modelo assistencial a partir da atenção básica(12).

\section{O Programa de Saúde da Família como Estratégia de Reorganização da Atenção à Saúde}

O PSF propõe a reorganização da atenção básica em ações de promoção da saúde, prevenção e riscos de doenças, resolutividade na assistência e recuperação, com Qualidade, o Que favorece a maior aproximação dos serviços à população( ${ }^{(2)}$.

A atenção primária é o "primeiro nível de contato", a porta de entrada dos indivíduos, das famílias e da comunidade no sistema para todas as novas necessidades e problemas. É uma abordagem Que forma a base e determina o trabalho de todos os outros níveis do sistema de saúde. Está atenta aos problemas mais comuns da comunidade, oferecendo serviços de prevenção, promoção, cura e reabilitação para maximizar a saúde e o bem estar ${ }^{(17)}$.

"... o conjunto de ações de saúde Que englobam a promoção, prevenção, diagnóstico, tratamento e reabilitação. É desenvolvido através do exercício de práticas gerenciais e sanitárias, democráticas e participativas, sob a forma de trabalho em equipe, dirigida a populações de territórios (território-processo) bem delimitados, pelas Quais assumem responsabilidade. Utiliza tecnologias de elevada complexidade e baixa densidade, Que devem resolver os problemas de saúde das populações de maior freeüência e relevância. É o contato preferencial dos usuários com o sistema de saúde. Orienta-se pelos princípios da universalidade, acessibilidade (ao sistema), continuidade, integralidade, responsabilização, humanização, vínculo, eQuidade e participação social. A atenção básica deve considerar o sujeito em sua singularidade, complexidade, inteireza e inserção sócio cultural, além de buscar a promoção de sua saúde, a prevenção e tratamento de doenças e a redução de danos ou de sofrimentos Que possam estar comprometendo sua possibilidade de viver de modo saudável”(18).

No Brasil, especialmente no âmbito do SUS, costuma-se referir à atenção primária enQuanto atenção básica. Esta elaboração conceitual no país, está relacionada à necessidade de construção de uma identidade institucional própria ${ }^{(18)}$, onde entende-se por atenção básica deve considerar como proposta da vigilância à saúde: o sujeito em sua singularidade, complexidade, inteireza e inserção sócio-cultural; o objeto da atenção Que apresenta uma dimensão coletiva (problemas de saúde de populações e potencialidades de organização social para a promoção da saúde), uma dimensão individual (sujeitos portadores de problemas, projetos e/ou sofrimentos), sendo Que o processo de trabalho da atenção básica deve se pautar no principio da integralidade ${ }^{(18)}$.

Neste sentido, o PSF destaca-se enQuanto estratégia inovadora e reestruturadora das ações e serviços de saúde, ao transpor a visão fragmentada do ser humano para uma compreensão integral na dimensão individual, familiar e coletiva, ou seja, o resgate da prática generalista, onde a compreensão do processo saúde doença a Que estão expostos os indivíduos passa a ser pensada de forma mais ampla, segundo a realidade local, comunitária e familiar ${ }^{(2)}$.

Para tanto, na reorganização das práticas de trabalho, tem-se como preceitos e atividades fundamentais, o diagnóstico de saúde da comunidade, o planejamento/programação locais, a complementaridade (integração entre os diversos níveis de atenção à saúde e sociossanitárias), a abordagem interprofissional, a referência e contra-referência, a educação continuada, a ação intersetorial, o acompanhamento/avaliação e o controle social ${ }^{(3)}$.

Nesse sentido, concebido inicialmente como um programa, é considerado pelo Ministério da Saúde como "uma estratégia estruturante dos sistemas municipais de saúde visando à reorientação do modelo de atenção e uma nova dinâmica da organização dos serviços e ações de saúde"(19).

Adota o princípio de adstrição de famílias (600 a 1000). correspondendo a 2.400 a 4.500 habitantes, sob a 
responsabilidade de uma equipe de saúde constituída por um médico, um enfermeiro, dois auxiliares de enfermagem e de Quatro a seis agentes comunitários de saúde ${ }^{(20)}$. Conjuga as ações de promoção, prevenção, diagnóstico precoce, tratamento e reabilitação, referenciando os casos de maior complexidade para os demais níveis de assistência ${ }^{(21)}$. Possibilita a integração e a promoção da organização das atividades em um território definido, com uma atuação inter e multidisciplinar ${ }^{(3,22)}$.

Além disso, incorpora e reafirma os princípios do SUS, tendo como meta ser substitutivo do modelo bio-médico hegemônico. Como forma de territorialização permite a demarcação de um espaço concreto de atuação da equipe de saúde, tendo o núcleo familiar como base e unidade para o desenvolvimento de sua atuação. Como orientador das políticas intersetoriais, tem papel fundamental, uma vez Que, pelas mesmas razões, é capaz de compreender que estruturas precisam ser modificadas visando ao bem estar da sua comunidade.

Da mesma forma, é capaz de ser importante mobilizador das forças sociais Que nesse espaço atuam, de modo a interagirem com o governo local na busca de soluções para os seus problemas $^{(18)}$. A execução das ações segue a lógica da vigilância à saúde e da valorização da relação com o usuário e a família( ${ }^{(1)}$.

\section{Desafios Apontados ao PSF na Reformulação do Modelo de Saúde}

O PSF vem se consolidando no Brasil, nos últimos anos tornouse a política de saúde prioritária do governo federal. Para tanto, faz-se necessário pontuar alguns aspectos relacionados a trajetória percorrida rumo a reorientação do modelo assistencial.

De acordo com dados do Ministério da Saúde de 2004, passados doze anos de implantação do PSF, são mais de 60 milhões de pessoas acompanhadas e 19.200 equipes na maior parte dos municípios brasileiros, com expressiva ampliação da oferta de serviços básicos em todo o país, abrindo acesso ao SUS a um grande segmento populacional, rural e urbano, antes excluído. Contudo, a Atenção Básica, com exceção de um número ainda inexpressivo de situações, pouco avançou na ampliação do tipo de ações ofertadas em cada Unidade Básica de Saúde (UBS), na Qualidade, no volume, na continuidade das ações e nos resultados à população, não alcançando o parâmetro universal de resolução de $90 \%$ dos problemas de saúde demandados neste nível ${ }^{(22)}$.

Neste sentido, Questiona-se por Que uma estratégia respaldada em um modelo teórico expressivo, capaz de modificar aspectos medulares importantes do sistema de saúde, não estaria sendo resolutiva.

A evidência científica demonstra Que a expansão do PSF tem favorecido a eqüidade e universalidade da assistência, uma vez Que as equipes têm sido implantadas, prioritariamente, em comunidades antes restritas Quanto ao acesso aos serviços de saúde. Entretanto, não se pode admitir, só pelas estatísticas, Que a integralidade das ações deixou de ser um problema na prestação da atenção, é necessário analisar os aspectos relacionados às práticas de saúde e aos processos de trabalho cotidiano ${ }^{(12)}$.

Desta forma, um dos desafios para implementação das diretrizes do PSF consiste em envolver os profissionais inseridos no programa em um amplo processo de reorientação do trabalho em saúde. Nesta Questão, devem-se levar em conta especificidades disciplinares - particularmente no Que se refere à racionalidade dominante em cada campo de conhecimento - às experiências de formação e à inserção profissional destes sujeitos, sem esQuecer a interferência do mercado neste processo ${ }^{(7)}$, Que supervaloriza o especialista e o desenvolvimento das especialidades médicas e facilita a penetração tecnológica interessado na capacidade lucrativa no setor saúde ${ }^{(23)}$.

Matumoto et $\mathrm{al}^{(7)}$ apontam Que grande parte dos médicos investigados não é egressa de cursos de pós-graduação em Saúde Coletiva ou, especificamente, Atenção Básica/Saúde da Família; são pediatras, generalistas e recém-formados. O perfil destes profissionais corresponde plenamente aos dados encontrados pelo estudo nacional ${ }^{(24,25)}$ sobre as equipes do PSF, destacando dois aspectos em particular: de um lado a residência e especialidades mais comuns dos médicos do PSF são as consideradas básicas: medicina interna, geral comunitária, pediatria e gineco-obstetrícia.; de outro, com relação ao tempo de experiência profissional destacam-se dois grupos com situações radicalmente opostas: recém formados Que ainda não têm carreiras consolidadas e para os Quais o PSF se apresenta como uma alternativa de "mercado de trabalho" e profissionais com larga trajetória profissional, incluindo aqui aposentados ou prestes a recorrer à aposentadoria.

A instabilidade do vínculo trabalhista e, conseQüentemente, a insegurança Que isto provoca no profissional, tem sido apontada como uns dos principais responsáveis pela alta rotatividade dos profissionais Que atuam nas equipes do PSF, prejudicando a continuidade e efetivação das ações em saúde ${ }^{(25,26)}$.

A formação do profissional capacitado para atuar no PSF e alocação destes pelos gestores de saúde nos serviços públicos é de alta importância, dada a abordagem Que este fará na reversão do modelo assistencial vigente, deslocando o foco de produção de procedimentos (consultas médicas, curativos, vacinas e outros) para a produção de cuidados $^{(6,7)}$. Mudanças curriculares na formação dos profissionais de saúde serão necessárias para transformação de uma visão flexneriana da saúde em uma proposta de produção de cuidados, acolhimento e humanização, Questões centrais no processo de reorientação do trabalho em saúde. Neste sentido, "os profissionais atualizam, projetam, estabelecem a oferta dos serviços de saúde a partir de uma concepção muito mais ampla, em Que trabalhar a doença não seja prioridade e em Que a promoção da saúde e a prevenção da doença proporcionam às comunidades ferramentas e tecnologias para sua participação no autocuidado"(27).

Centrar na lógica da produção do cuidado é outro desafio para o programa, visto Que se traduz no trabalho orientado aos problemas, às necessidades e à Qualidade de vida do usuário. São ações Que, além de produzir os procedimentos inerentes ao caso, como uma sutura, um curativo, uma consulta centram, nas relações humanas, na produção de vinculo, no acolhimento ${ }^{(6,7)}$.

De acordo com Franco et $\mathrm{al}^{(28)}$, o acolhimento propõe Que o serviço de saúde seja organizado de forma usuário-centrada, partindo dos seguintes princípios: garantir a acessibilidade universal a partir do atendimento de todas as pessoas; reorganizar o processo de trabalho, afim de que se desloque o eixo central do médico para uma equipe multiprofissional (equipe de acolhimento), Qualificando a relação trabalhador-usuário, Que deve dar-se por parâmetros humanitários de solidariedade e cidadania. Somados 
a isso, se acrescenta Que o acolhimento propõe, principalmente, reorganizar o serviço, no sentido da garantia no acesso universal, resolubilidade e atendimento humanizado.

No entanto, Quando se trabalha sob a ótica do modelo médicocentrado, o trabalho se organiza para atuar sobre problemas específicos por meio do atendimento do médico, subordinando os saberes e ações dos outros profissionais à lógica médica, diminuindo, assim, o espaço da dimensão cuidadora da equipe, empobrecendo a possibilidade de incorporação de outros saberes para ampliação da ação clínica, e o campo de busca para a solução dos problemas ${ }^{(7)}$.

O trabalho em equipe também apresenta-se com grandes limitações. Estudos recentes apontados por Silva et $\mathrm{al}^{(21)}$ sobre o trabalho da equipe multiprofissional no PSF revelaram: ausência de responsabilidade coletiva do trabalho e baixo grau de interação entre as categorias profissionais, onde apesar do discurso de teor igualitário, os membros das equipes de saúde da família mantêm representações sobre hierarQuia entre profissionais e não profissionais, nível superior e nível médio de educação, médico e enfermeiro.

Um ponto de tensão apresentado por Franco et al ${ }^{(28)}$ está relacionado ao trabalho nos estabelecimentos de saúde organizado, tradicionalmente de forma parcelado. Em eixo verticalizado, organiza-se o trabalho do médico e entre estes de cada especialidade médica e assim, sucessivamente, vai se organizando o trabalho dos outros profissionais. A superespecialização e o trabalho fracionado fazem com Que o profissional de saúde se aliene do próprio objeto de trabalho, no caso do médico, este não consegue romper com a lógica do trabalho médico, Que se dá em torno da agenda/consulta e com isso a peQuena inserção dos profissionais médicos no acolhimento. Para solucionar este problema os autores consideram o vínculo como a diretriz Que, acoplada ao acolhimento é capaz de garantir o real reordenamento do processo de trabalho na UBS.

Outro desafio apontado pela fragmentação do trabalho é a prática da intersetorialidade. Segundo Paula et al ${ }^{(29)}$, percebe-se, nos depoimentos dos entrevistados, uma confusão conceitual sobre intersetorialidade, pois tomam-na como responsabilidade individualizada a partir das demandas individualizadas dos usuários. Desta forma, se identifica a intersetorialidade como um processo de trabalho penoso e solitário. Entende-se, ainda, a necessidade da criação de uma rede de apoio para a efetivação desse princípio e acredita-se que é preciso começar a entender e escutar a opinião de outros profissionais e setores.

Um obstáculo apresentado por Alves ${ }^{(12)}$ e Berni et $\mathrm{al}^{(30)}$, diz respeito às práticas de educação em saúde. Tais autores retratam Que tais práticas estão baseadas no modelo tradicional hegemônico Que privilegia a assistência curativa e preconiza Que a prevenção das doenças prima pela mudança de atitudes e comportamentos individuais, nas estratégias desta prática incluem informações verticalizadas Que ditam comportamentos a serem adotados para a manutenção da saúde. Berni et al ${ }^{(30)}$ enfatiza Que a participação ativa da população nas decisões de cuidado com o corpo não são práticas presentes no cotidiano dos serviços de saúde.

De acordo com esta premissa, os usuários são tomados como indivíduos carentes de informação em saúde mantendo uma relação assimétrica entre profissionais e usuários, uma vez Que um detém um saber técnico-científico, com status de verdade, enquanto o outro precisa ser devidamente informado. Além de reagir a um estimulo apenas temporário, a população não muda de comportamento e gera a falta de estímulo na organização da comunidade para o desenvolvimento de cidadania e participação nas atividades desenvolvidas pela unidade.

Sendo o PSF um "lócus" de fomento à participação da população, deve promover senão uma nova relação entre os sujeitos, onde tanto o profissional Quanto o usuário podem ser produtores de saúde ${ }^{(6)}$. Este envolvimento só é possível mediante um processo de diálogo entre os saberes, no Qual um contribui com seu conhecimento peculiar da situação, acreditando-se Que o desenvolvimento efetivo dessa participação parte de práticas vivenciadas no cotidiano.

Feuerwerker ${ }^{(32)}$ afirma Que o desafio na tendência de mudança do modelo de atenção se dá pelo fato do modelo estar em construção envolvendo Questões Que não são simples, como as articulações clínica e saúde coletiva e o trabalho multiprofissional. Mas vê também uma oportunidade de repensar a formação médica e certamente de outros profissionais de saúde, Quando se abre demanda para um novo perfil de profissional de saúde direcionado a um modelo holístico e mais humanizado Que impacte mais positivamente sobre os indicadores sanitários e Que, ao mesmo tempo trabalhem mais contentes e profissionalmente mais realizados.

Remodelar a assistência à saúde implica modificar o processo de trabalho e estas são Questões Que foram colocados em pauta a partir da implantação e construção do PSF no cotidiano dos municípios e localidade. É fato Que a sua implantação por si só não garante Que o modelo assistencial esteja sendo modificado, mas com certeza já nos leva a refletir sobre mudanças necessárias e urgentes na forma de agir e implementar ações e serviços no contexto da Estratégia de Saúde da família.

\section{CONCLUSÃO}

Apresentar o PSF, como uma proposta de reorientação do cuidado à saúde, é enfrentar o desafio de construir novas bases para o desenvolvimento de novas práticas sanitárias. É colocar um desafio ainda maior de desconstruir as matrizes de um modelo assistencial perverso, sob os Quais, trabalhadores e gestores de saúde, governantes e a própria população estão inseridos. É colocar em pauta na agenda da saúde da população brasileira conceitos como: vínculo, acolhimento e cuidado no contexto de uma atenção sanitária humanizada e humanística.

Este desafio de desconstrução implica muito labor, mas a vantagem da superação destes desafios leva a transformação da realidade e à construção de práticas de saúde solidárias, acolhedoras e conseqüentemente mais efetivas e resolutivas.

O direito universal à saúde conQuistado pela Constituição Federal Brasileira de 1988 e a criação do PSF pelo MA em 1994 colocam a promoção da Saúde e a prevenção da enfermidade definitivamente na pauta da agenda nacional. A estratégia seguinte seria a construção de um trabalho conjunto da universalidade com os serviços de saúde e a comunidade, criando espaços de parceria Que fortaleçam o aparecimento de novas formulações sobre o pensar e o fazer sanitários. 


\section{REFERÊNCIAS}

I. Campos CEA. O desafio da integralidade segundo as perspectivas da Vigilância da Saúde e da Saúde da Família. Rev Ciência Saúde Coletiva 2003; 8(2): 569-84.

2. Fernandes AS, Seclen-Palacin I. Experiências e desafios da atenção básica e saúde da família: caso Brasil. Brasília: OPAS; 2004.

3. Ministério da Saúde (BR). Manual para organização da atenção básica. Brasília: Ministério da Saúde; 1998.

4. Cotta RMM, Mendes FF, Muniz IN. Descentralização das políticas públicas de saúde - do imaginário ao real. Viçosa: UFV - Cebes; 1998.

5. Cotta RMM, Morales MSV, Cotta Filho IS, Gonzáles AL, Ricos IAD. Obstáculos e desafios da saúde pública no Brasil. Rev Hospital Clínicas Porto Alegre 2002; 22(1): 25-32.

6. Merhy EM. Introdução ao debate sobre os componentes da caixa de ferramentas dos gestores em saúde. Rev SUS. Brasil - vivências e estágios na realidade do SUS do Brasil. Disponível em: http://dtr2002.saude.gov.br/versus/index.html

7. Matumoto S, Fortuna CM, Mishima SM, Pereira MJB, Domingos NAM. Supervisão de equipes no Programa de Saúde da Família: reflexões acerca do desafio da produção de cuidados. Rev Interface-Comun Saúde Educ 2005; 8(16): 9-24.

8. Ministério da Saúde (BR). O desenvolvimento do Sistema Único de Saúde: avanços, desafios e rearfirmação dos seus princípios e diretrizes. Brasília: Ministério da Saúde; 2002.

9. Ministério da Saúde (BR). Conselho nacional de saúde. Para entender a gestão do SUS. Brasília: Ministério da Saúde; 2003.

10. Mendes EV. Uma agenda para a saúde. São Paulo: Hucitec. 1996.

I I. Scherer MDA, Marino RSA, Ramos FRS. Rupturas e resoluções no modelo de atenção à saúde: reflexões sobre a estratégia saúde da família com base nas categorias kuhnianas. Rev Interface-Comun Saúde Educ 2005; 9(I6): 53-66.

12. Alves VS. Um modelo de educação em saúde para o Programa Saúde da Família: pela integralidade da atenção e reorientação do modelo assistencial. Rev Interface-Comun Saúde Educ 2005; 9 ( 16 ): 39-52.

13. Czeresnia D. O conceito de saúde e a diferença entre prevenção e promoção. In: czeresnia D, Freitas CM, organizadores. Promoção da saúde: conceitos, reflexões, tendências. Rio de Janeiro: Fiocruz; 2003. p. 39-5 I.

14. Canguilhem GO. Normal e patológico. In: Czeresnia D, Freitas CM, organizadores. Promoção da saúde: conceitos, reflexões, tendências. Rio de laneiro: Fiocruz; 2003. p.39-51.

15. Paim IS. Vigilância a Saúde: dos modelos assistenciais para a promoção da saúde. In: Czeresnia D, Freitas CM, organizadores. Promoção da saúde: conceitos, reflexões, tendências. Rio de Janeiro: Fiocruz; 2003. p.161-71.

16. Teixeira CF, Paim IS, Vilasbôas AL. SUS, modelos assistenciais e Vigilância de Saúde. Infor Epidemiol SUS 1998; 7(2): 7 28
17. Starfield B. Atenção primaria - equilíbrio entre necessidades de saúde, serviços e tecnologia. Brasília: Ministério da Saúde: 2004.

18. Ministério da Saúde (BR). Departamento de atenção básica. Documento final da comissão de avaliação da atenção básica. Produto do trabalho da comissão instituída pela portaria $\mathrm{n}^{\circ}$ $676 \mathrm{gm} / \mathrm{ms}$ de 03 de junho de 2003. Diário Oficial da União 2003 jun 4

19. Ministério da Saúde (BR). Monitoramento da implantação e desenvolvimento das equipes de Saúde da Família no Brasil. Brasília: MInistério da Saúde; 2002.

20. Ministério da Saúde (BR). Guia Prático Saúde da Família. Brasília: Ministério da Saúde; 200 I.

21. Silva IZQI. O trabalho em equipe no PSF: investigando a articulação técnica e a interação entre os profissionais. Rev Interface-Comun Saúde Educ 2005; 9(16): 28.

22. Ministério da Saúde (BR). Informe da Atenção Básica. Brasília: Ministério da Saúde; 2004;

23. Lampert JB. Currículo de graduação e o contexto da formação do médico. Rev Bras Educ Méd 200 I; 25(1): 7-19.

24. Machado MH. Perfil dos médicos e enfermeiros do programa saúde da família. Brasília: MS/Fiocruz; 2000.

25. Meneses e Rocha AAR. A trajetória profissional de cinco médicos do Programa de Saúde da Família: os desafios de construção de uma nova prática. Rev Interface-Comun Saúde Educ 2005; 9(17): 303-16.

26. Dal Poz MR. Câmbios em la contratación de recursos humanos: el caso del programa de salud de la família em Brasil. Gac Sanit Espanha 2002; 16(1): 82-86.

27. Almeida M, Feuerwerker L, Llanos M, organizadores. A educação dos profissionais de saúde na américa latina: teoria e prática de um movimento de mudança. São Paulo: Hucitec; 1999.

28. Franco TB, Bueno WS, Merhy EE. O acolhimento e os processos de trabalho em saúde: o caso de Betim, Minas Gerais, Brasil. Cad Saúde Pública 1999; 15(2): 345-53.

29. Paula KA, Palha PF, Protti ST. Intersetorialidade uma vivencia pratica ou um desafio a ser conQuistado? O discurso do sujeito coletivo dos enfermeiros nos núcleos de saúde da família do distrito oeste- Ribeirão Preto. Rev Interface-Comunic Saúde Educ; 2004; 8(15): 331-48.

30. Berni NIO, Luz MH, Kohirausch SC. Conhecimento, percepções e assistência à saúde da mulher no climatério. Rev Bras Enfermagem 2007; 60(3): 299-306.

31. Yepez MT, Morais NA. Reivindicando a subjetividade dos usuários da Rede Básica de Saúde: para uma humanização do atendimento. Cad Saúde Publica 2004; 20(I): 80-8.

32. Feuerwerker LCM, Sena RR. A construção de novos modelos acadêmicos, de atenção à saúde e de participação social. In: Lampert JB. Currículo de graduação e o contexto da formação do médico. Rev Bras Educ Méd 200 I ; 25(1): 7-19. 